\title{
Inguinoscrotal bladder hernias: report of a series and review of the literature
}

\author{
Kate H. Kraft, MD, ${ }^{*}$ Sarah Sweeney, BSc, ${ }^{\dagger}$ Aaron S. Fink, MD, ${ }^{\ddagger}$ Chad W.M. Ritenour, MD, ${ }^{\xi}$ \\ Muta M. Issa, MD, MBA"
}

\begin{abstract}
Bladder involvement occurs in $1 \%-4 \%$ of cases of inguinal hernias. Among obese men aged 50 to 70 , the incidence may reach $10 \%{ }^{1,2}$ The diagnosis of bladder involvement is often difficult to delineate at the time of presentation and may only become apparent at the time of herniorrhaphy. Surgical management pertaining to the approach, repair and potential need for bladder resection may challenge the surgeon. We report a series of 4 cases of large inguinoscrotal bladder hernias and provide a literature review. Our goal is to highlight the clinical presentation and the decisive issues surrounding the diagnosis and management of this condition.
\end{abstract}

Can Urol Assoc J 2008;2(6):619-23

\section{Introduction}

About $1 \%-4 \%$ of all inguinal hernias involve the bladder, and the incidence may reach $10 \%$ among obese men aged 50 to 70 years. ${ }^{1,2}$ Most cases are asymptomatic and are usually found incidentally on radiographic imaging or at the time of herniorrhaphy. Occasionally, the herniated bladder becomes entrapped within the inguinal canal and makes its way into the scrotum. In such advanced cases, the patient typically completes 2 -stage urination (manually compresses his scrotum for complete bladder emptying). ${ }^{1-3}$ Inguinoscrotal bladder hernias have also been associated with severe complications, including urinary tract infections, obstructive uropathy and bladder infarctions requiring subtotal cystectomy. ${ }^{1,4,5}$ We report a series of 4 cases of large inguinoscrotal bladder hernias (Table 1) and provide a brief literature review.

\section{Case reports}

\section{Case \# 1}

A 71-year-old white man presented with a 6-month history of intermittent gross hematuria, irritative lower urinary tract symptoms (LUTS) and urinary incontinence. He also had a history of transitional cell carcinoma of the bladder treated successfully with transurethral resection and intravesical immunotherapy 15 years before presentation. Additional comorbidities included significant chronic obstructive pulmonary disease that required home oxygen. A large $(10-\mathrm{cm})$ right inguinal hernia extending into the right hemiscrotum (Fig. 1A) and an enlarged prostate consistent with benign prostate hyperplasia $(\mathrm{BPH})$ were noted on physical examination. Urine culture showed Klebsiella oxytoca $(10000-50000 \mathrm{cfu} / \mathrm{mL})$ which was subsequently treated effectively with antibiotics. Renal function (creatinine $70 \mu \mathrm{mol} / \mathrm{L}$ ) and age-specific serum prostate-specific antigen (PSA) level $(3.6 \mu \mathrm{g} / \mathrm{L})$ were normal.

Cystoscopy and cystography revealed a mildly trabeculated bladder with herniation via the right inguinal canal into the right hemiscrotum (Fig. 2). There was no evidence of suspicious bladder lesions. Bladder wash cytology was negative for malignant cells.

The patient opted to pursue surgical repair. He was given local anesthesia block because he had pre-existing pulmonary disease. His right groin was explored, revealing herniation of the bladder through the right inguinal canal into the hemiscrotum. The herniated bladder was freed from the structures within the inguinal canal and the hemiscrotum, then returned to its normal pelvic position. The inguinal floor was repaired using a modified Bassini technique.

The patient's postoperative recovery was uneventful, and his hernia symptoms resolved. Postoperative cystogram demonstrated a normal bladder. The patient had residual $\mathrm{BPH}$ symptoms, which were managed with medical therapy and intermittent selfcatheterization. He responded favourably and was pleased with the result of his urological treatment (Fig. 1B).

\section{Case \# 2}

A 78-year-old white man presented with recurrent right flank pain, moderate LUTS (International prostate symptom score [IPSS] 16/35) and intermittent swelling in the right groin and hemiscrotum. He had undergone 
a previous right inguinal hernia repair several years before presentation. Occasionally, he had to squeeze his scrotum to complete urination. His past medical history was significant for hyperlipidemia, hypertension, and cardiac arrhythmias requiring pacemaker implantation.

Physical examination revealed no flank mass or tenderness. A 7-cm reducible right inguinal hernia extending into the right hemiscrotum was noted. The prostate was enlarged. The serum creatinine level was $132 \mu \mathrm{mol} / \mathrm{L}$ and the serum PSA level was $0.94 \mu \mathrm{g} / \mathrm{L}$. Urinalysis and urine culture were negative.

\begin{tabular}{lcccc}
\hline Table 1. Summary of cases & \multicolumn{4}{c}{ Case } \\
& \multicolumn{5}{c}{. } \\
\cline { 2 - 5 } Characteristic & 1 & 2 & 3 & 4 \\
\hline Age, yr & 71 & 78 & 55 & 58 \\
Hernia size, cm & 10 & 15 & 6 & 7 \\
Location & Right & Right & Right & Right \\
IPSS & NA & 16 & 28 & 21 \\
2-stage urination & $\sqrt{ }$ & $\sqrt{ }$ & $\sqrt{ }$ & None \\
Gross hematuria & $\sqrt{ }$ & None & None & $\sqrt{ }$ \\
Enlarged prostate & $\sqrt{ }$ & $\sqrt{ }$ & $\sqrt{ }$ & $\sqrt{ }$ \\
Cystoscopy & $\sqrt{ }$ & $\sqrt{ }$ & $\sqrt{ }$ & $\sqrt{ }$ \\
CT scan & NA & Positive & Positive & Positive \\
Hernia repair & $\sqrt{ }$ & $\sqrt{ }$ & $\sqrt{ } *$ & $\sqrt{ }$ \\
\hline CT $=$ computed tomography; IPSS $=$ International Prostate Symptom Score; NA $=$ data not \\
available. \\
${ }^{*}$ Patient underwent emergent inguinal hernia repair at an outside facility. & \\
\hline
\end{tabular}

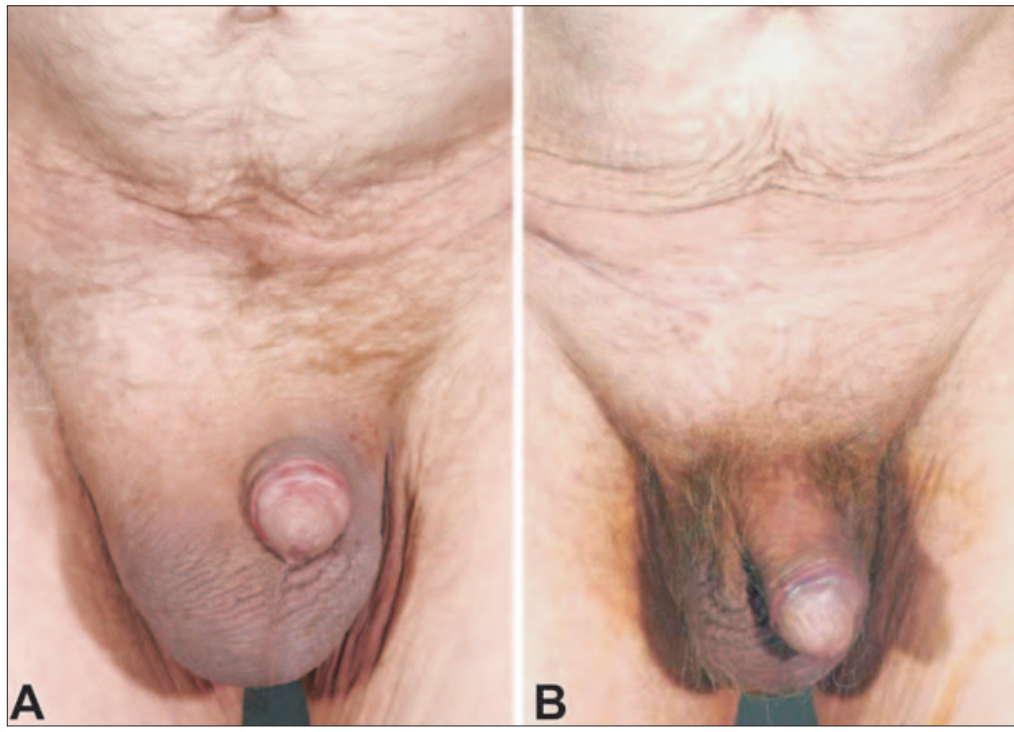

Fig. 1. Preoperative appearance of right inguinal hernia in patient 1 (A) and result after surgical repair (B).
An intravenous urogram (IVU) and computed tomography (CT) scan demonstrated the right side of the bladder wall herniating through the right inguinal canal into the right hemiscrotum (Fig. 3 and Fig. 4). Cystoscopy and cystography confirmed the radiological findings, with grade II trabeculation of the bladder and no evidence of urothelial lesions.

The patient underwent surgical exploration of the right groin under general anesthesia. The herniated bladder was dissected from the inguinal canal and the hemiscrotum, and returned to its normal pelvic position. The inguinal floor was repaired using a modified Bassini technique. Postoperative recovery was uneventful and there were no complications. The patient's preoperative symptoms resolved. Postoperative cystography demonstrated a normal bladder.

\section{Case \# 3}

A 55-year-old black man presented with severe and

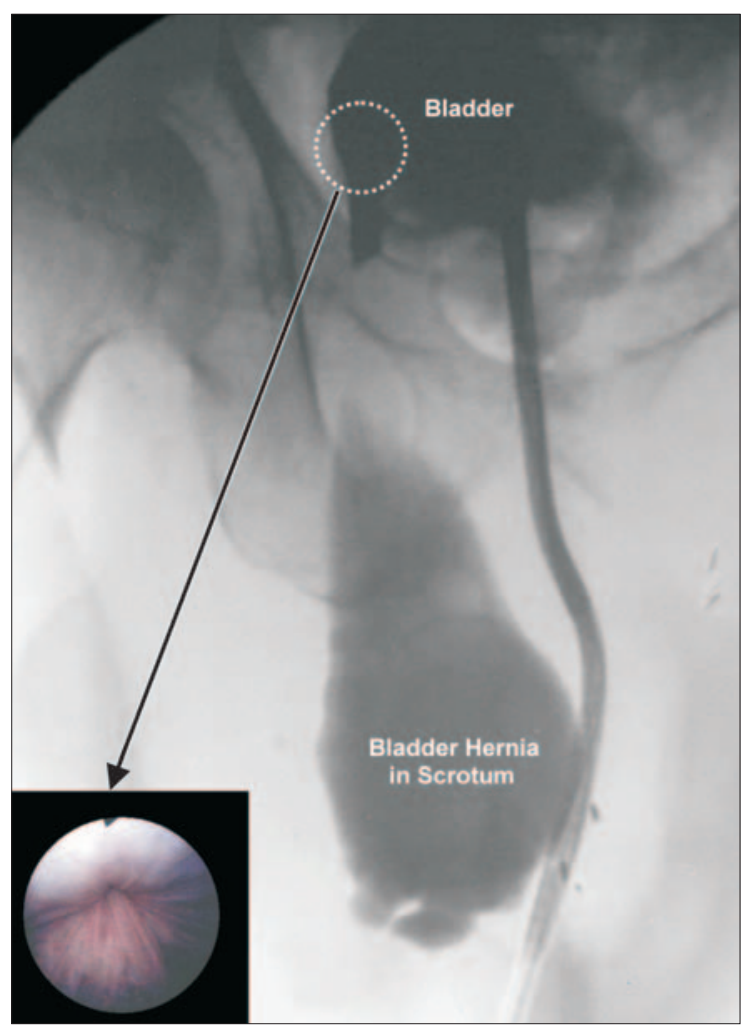

Fig. 2. Cystogram showing the bladder of patient 1 herniating through the right inguinal canal into the right hemiscrotum. The insert shows the cystoscopic appearance of the bladder as it entered the inguinal canal. 
bothersome LUTS (IPSS 28/35) and intermittent swelling in the right scrotum. The patient had to squeeze his scrotum to complete urination. He had significant medical comorbidities including congestive heart failure, hypertension, gastroesophageal reflux with hiatal hernia, and obesity. A large $(15-\mathrm{cm})$ irreducible right inguinal hernia extending into the right hemiscrotum was noted on physical examination. Serum creatinine was $114 \mu \mathrm{mol} / \mathrm{L}$ and serum PSA was $1.85 \mu \mathrm{g} / \mathrm{L}$. Urinalysis and urine culture were negative. Scrotal ultrasound findings were consistent with a right inguinal hernia extending into the right hemiscrotum.

Cystoscopy and cystography revealed herniation of the right side of the bladder through the right inguinal canal into the right hemiscrotum. The prostate appeared enlarged. There was no evidence of bladder trabeculation or urothelial lesions.

The patient was scheduled for inguinal exploration and surgical hernia repair. In the interval, he presented to an outside hospital with acute pain/incarceration of his hernia and underwent surgical treatment by an outside surgeon. Followup evaluation 6 months postoperatively confirmed successful results and complete resolution of hernia symptoms.

\section{Case \# 4}

A 58-year-old white man presented with right inguinal swelling, intermittent gross hematuria and moderate LUTS (IPSS 21/35). He stated that "my bladder goes back to the right place when I wake up in the morning." Other illnesses included diabetes mellitus and hyperlipidemia.

A 6-cm reducible right inguinal hernia extending into the right hemiscrotum was noted on physical examination. Serum creatinine was $114 \mu \mathrm{mol} / \mathrm{L}$ and serum PSA was $0.22 \mu \mathrm{g} / \mathrm{L}$. Urinalysis and urine culture were negative. CT scan revealed the right side of the bladder wall herniating through the right inguinal canal into the right hemiscrotum. Cystoscopy and cystography confirmed the radiological findings. The prostate appeared enlarged and the bladder had grade IV trabeculation without urothelial lesions. The patient underwent surgical exploration and right inguinal hernia repair using mesh. Postoperative recovery was uneventful, and the patient's hernia symptoms resolved.

\section{Discussion}

About $1 \%-4 \%$ of all inguinal hernias involve the

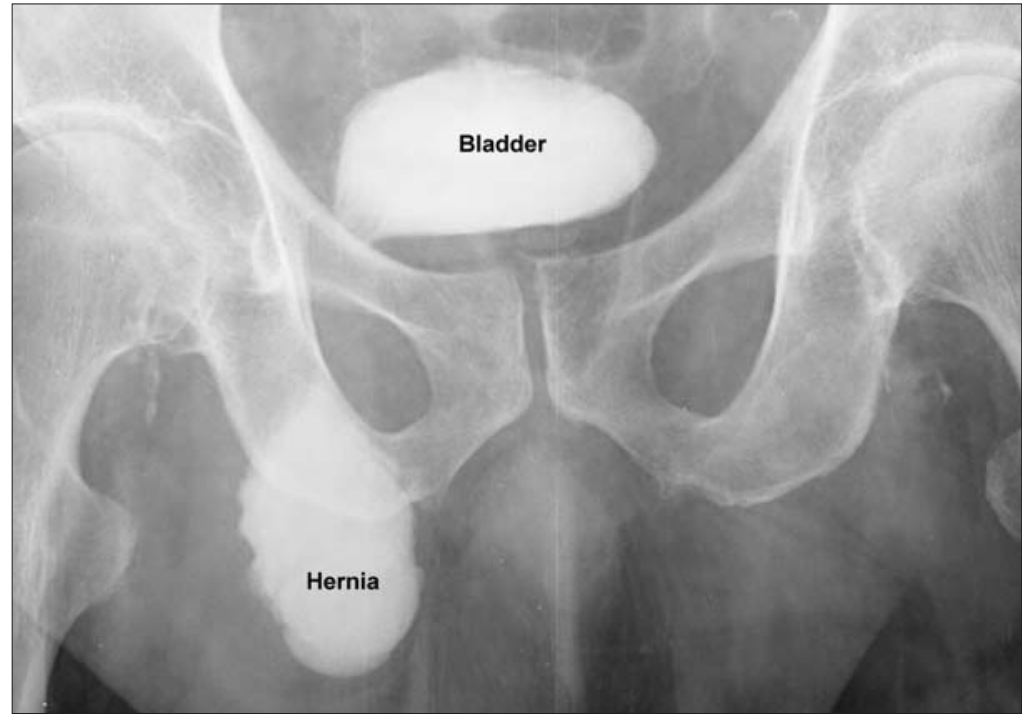

Fig. 3. An intravenous urogram showing the bladder of patient 2 herniating through the right inguinal canal into the right hemiscrotum.

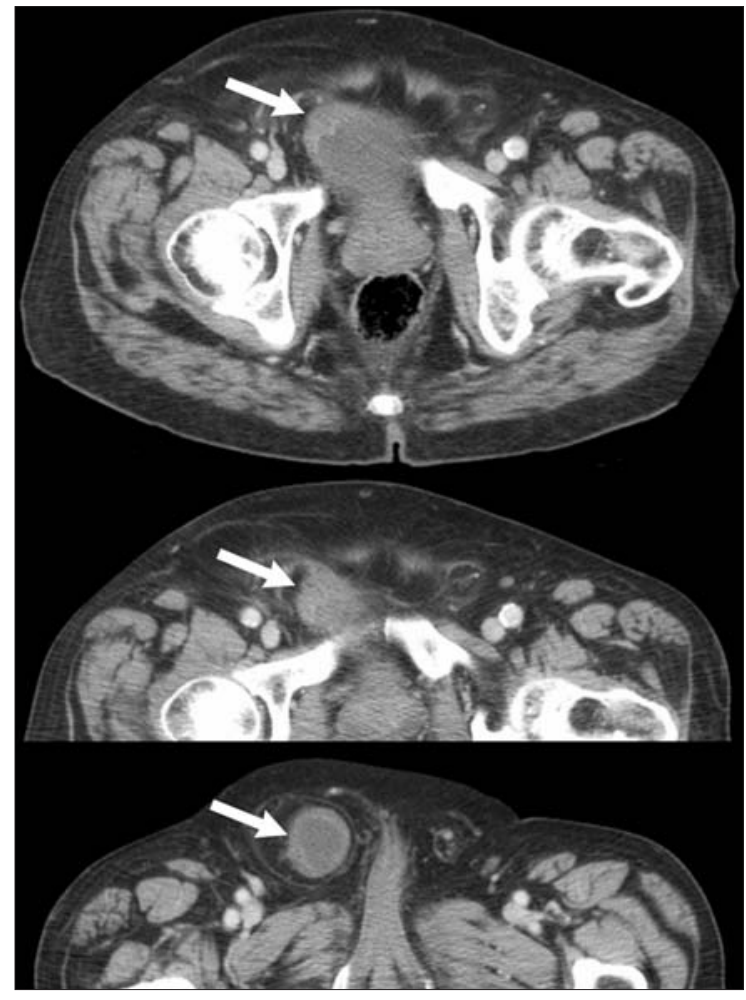

Fig. 4. Serial computed tomography images showing the bladder of patient 2 herniating into the right inguinal canal. The arrow identifies the bladder surrounded by the spermatic cord within the lower part of the inguinal canal. 
Kraft et al.

bladder, which may increase the risk of complications during surgical repair. Inguinoscrotal bladder hernias usually present with intermittent swelling in the groin/scrotum and significant LUTS, as seen in all 4 of our patients. More advanced cases may be associated with 2-stage urination or a decrease in scrotal size after voiding. ${ }^{6} \mathrm{All} 4 \mathrm{pa}-$ tients in our relatively small series experienced such 2 -stage urination. Although our series is small, it does highlight 2-stage urination to be a common symptom in patients with bladder hernias. Associated pathologies include BPH, bilateral hydronephrosis with or without acute renal failure, stones within the herniated bladder, vesicoureteral reflux, necrosis of the bladder and scrotal abscess. Among previously cited cases, the rate of these comorbidities is about $24 \% .^{7}$ In addition, patients with bladder hernias have demonstrated a higher incidence of genitourinary cancers. Oruç and colleagues ${ }^{17}$ review revealed 13 cases with malignancy out of 116 patients with inguinoscrotal bladder herniation $(11 \%)$. Nine were bladder carcinoma, 3 were prostate carcinoma and 1 was reported as a neoplasm.

Bladder involvement in inguinal hernias is often not recognized before surgical repair. Less than $7 \%$ of bladder hernias are diagnosed preoperatively, $16 \%$ are diagnosed postoperatively because of complications and the remainder of cases are diagnosed perioperatively. ${ }^{8}$ The risk of bladder injury during herniorrhaphy has been reported as $12 \%{ }^{9}$ Therefore, accurate diagnosis of inguinoscrotal bladder hernias is important to avoid injury to the bladder during surgical repair. ${ }^{7,9,10}$

Radiological investigations include CT scan, IVU and/or cystogram. These are equally successful in establishing the diagnosis, as demonstrated in our series. Ultrasonography may also be used and can differentiate the bladder from other intrascrotal conditions such as hydrocele, spermatocele, epididymal cyst and abscess. ${ }^{9}$ In one report, ultrasonography is advocated when the diagnosis of a bladder hernia is suspected clinically (e.g., 2-stage urination), when IVU demonstrates suggestive findings or when inguinal hernia repair is considered in older men with significant LUTS. ${ }^{3}$

Flexible cystoscopy is indicated to confirm the diagnosis, evaluate the prostate and the bladder, and exclude additional pathology in cases with gross hematuria. The radiological and cystoscopic appearance of the bladder hernia typically mimics that of a bladder diverticulum. The urologist should be aware of this in planning the appropriate surgical approach. An abdominal surgical approach with an attempt to resect the bladder "diverticulum" is unnecessary and would subject the patient to additional risks and potential complications.

The standard treatment for inguinal hernias involving the bladder is surgical repair (herniorrhaphy). On surgical exploration of the inguinal canal, the bladder is identified, dissected off its surrounding structures and reduced to its original location within the pelvis. Standard repair of the direct defect in the inguinal floor is then performed according to the surgeon's preference. Historically, surgeons resected the herniated portion of the bladder when the hernia was massive. Current recommendations suggest bladder resection should be reserved only for cases with bladder wall necrosis, true herniated bladder diverticulum, a tight hernial neck or tumour in the herniated bladder..$^{5,6}$

Patients with significant comorbidities and high surgical risk may be offered standard inguinal hernia repair. The procedure can be performed safely under local anesthesia as was done for one of our patients. Due to the potential perioperative complications, we recommend reserving this for patients who are significantly symptomatic and bothered by their symptoms.

Occasionally, patients may opt for conservative therapy (watchful waiting). Other conservative management includes intermittent urethral catheterization to help reduce the bladder out of the hernia sac. ${ }^{3}$

Prostate enlargement and inguinal hernias often coexist in the elderly male population, as in all 4 of our patients. Difficulty arises in determining whether LUTS are caused by the entrapped herniated bladder, enlarged prostate or both. Historically, inguinal hernia repair was not recommended until BPH had been treated surgically, assuming that LUTS and straining might adversely affect the hernia repair and risk recurrence. In the modern era of medical BPH therapy, we would recommend re-evaluating the patient's symptoms following medical BPH therapy $(\alpha$-blocker and $5 \alpha$-reductase inhibitors) and inguinal hernia repair. Transurethral resection of the prostate may prove challenging in patients with large bladder hernias owing to entrapment of irrigation fluid and prostatic chips within the herniated segment of the 
bladder. The procedure may prove unnecessary if the patient's LUTS resolve following the inguinal hernia repair. All 4 patients in our series had their LUTS resolve within a few months after the hernia repair, that is, no patient required surgical intervention for prostate enlargement. This suggests that a large component of the LUTS was related to their inguinoscrotal bladder hernia.

\section{Conclusion}

In summary, inguinoscrotal bladder hernias often go unrecognized preoperatively. Accurate diagnosis can be readily established radiologically and/or with cystoscopy. Surgical repair through an inguinal approach is the preferred treatment and can be performed under local anesthesia in patients at high risk for general anesthesia. Resection of bladder tissue is rarely indicated. Concomitant enlargement of the prostate should be treated medically first and re-evaluated after inguinal hernia repair.

*Resident in Urology, †Research Assistant in Urology, ‡Professor of Surgery, §Assistant Professor of Urology, 7 Associate Professor of Urology, Department of Urology, Atlanta Veterans Affairs Medical Center and Emory University School of Medicine, Atlanta, Ga.
This article has been peer reviewed.

Competing interests: None declared.

\section{References}

1. Fisher PC, Hollenbeck BK, Montgomery JS, et al. Inguinal bladder hernia masking bowel ischemia. Urology 2004;63:175-6.

2. Huerta $S$, Fairbanks $T$, Cinat $M$. Incarcerated vesicoinguinal hernia presenting with gross hematuria. J Am Coll Surg 2005;201:992-3.

3. Shelef I, Farber B, Hertzanu Y. Massive bladder hernia: ultrasonographic imaging in two cases. Br J Urol 1998;81:492-3.

4. Laniewski PJ, Watters GR, Tomlinson P. Herniation of the bladder trigone into an inguinal hernia causing acute urinary obstruction and acute renal failure. J Urol 1996;156: 1438-9.

5. Vindlacheruvu RR, Zayyan K, Burgess NA, et al. Extensive bladder infarction in a strangulated inguinal hernia. Br J Urol 1996;77:926-7.

6. Wagner AA, Arcand P, Bamberger MH. Acute renal failure resulting from huge inguinal bladder hernia. Urology 2004;64:156-7.

7. Oruç MT, Akbulut $Z$, Ozozan 0 , et al. Urological findings in inguinal hernias: a case report and review of the literature. Hernia 2004;8:76-9.

8. Watson LF. Hernia. 3rd ed. St. Louis (MO): CV Mosby, 1948.

9. Catalano 0 . Ultrasound evaluation of inguinoscrotal bladder hernias: report of three cases. Clin Imaging 1997;21:126-8.

10. Gomella $L G$, Spires $S M$, Burton JM, et al. The surgical implications of herniation of the urinary bladder. Arch Surg 1985; 120:964-7.

Correspondence: Dr. Muta M. Issa, Department of Urology (112), Atlanta Veterans Affairs Medical Center, 1670 Clairmont Rd., Decatur GA 30033; fax 404 3292201; issa@emory.edu

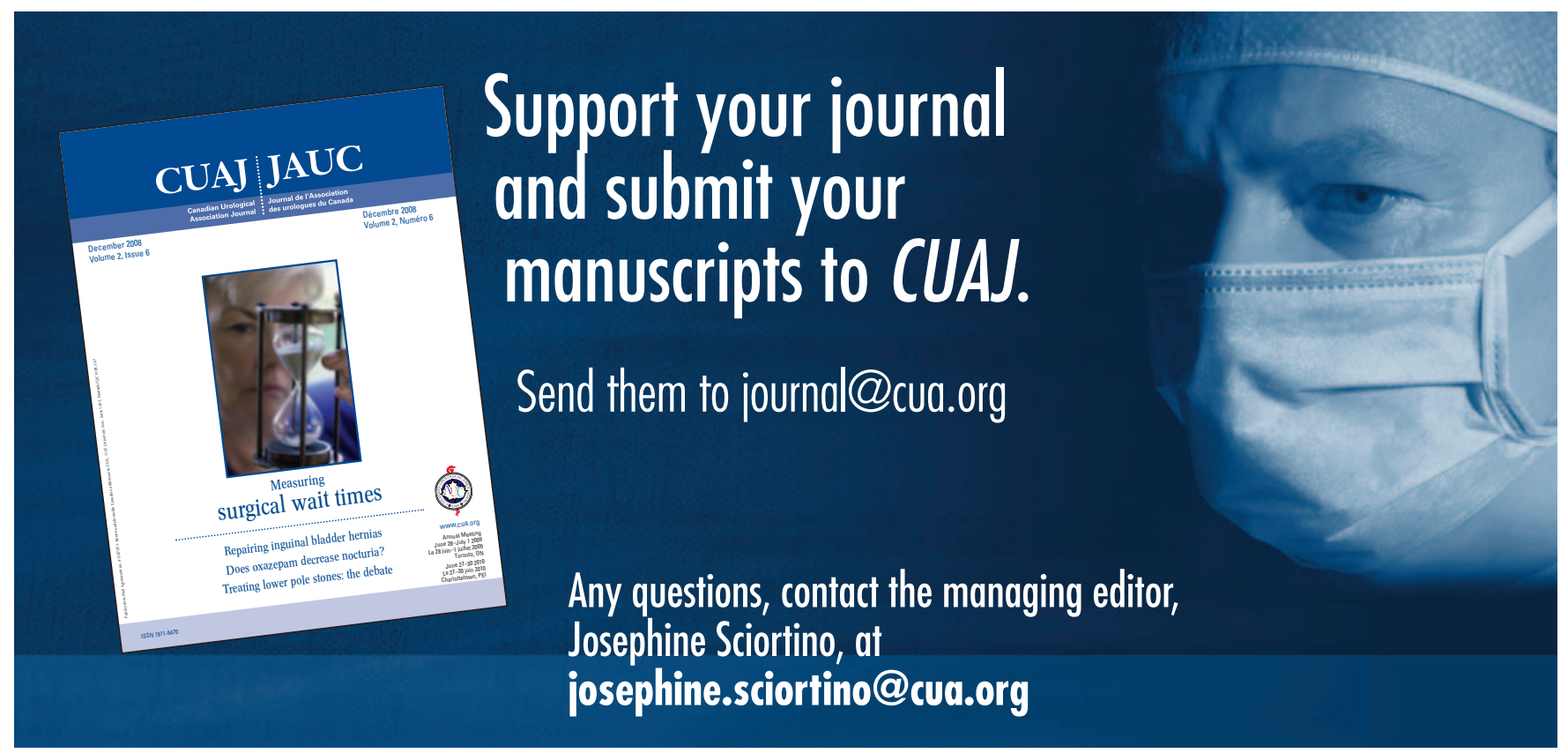

